\title{
Prevention of murine autoimmune diabetes by CCL22-mediated Treg recruitment to the pancreatic islets
}

\author{
Joel Montane, ${ }^{1}$ Loraine Bischoff, ${ }^{1}$ Galina Soukhatcheva, ${ }^{1}$ Derek L. Dai, ${ }^{1}$ Gijs Hardenberg, ${ }^{2}$ \\ Megan K. Levings, ${ }^{2}$ Paul C. Orban, ${ }^{1}$ Timothy J. Kieffer, ${ }^{2,3}$ Rusung Tan, ${ }^{1}$ and C. Bruce Verchere ${ }^{1,2}$ \\ ${ }^{1}$ Departments of Pathology and Laboratory Medicine, Child and Family Research Institute, ${ }^{2}$ Department of Surgery, and \\ ${ }^{3}$ Department of Cellular and Physiological Sciences, University of British Columbia, Vancouver, British Columbia, Canada.
}

\begin{abstract}
Type 1 diabetes is characterized by destruction of insulin-producing $\beta$ cells in the pancreatic islets by effector $T$ cells. Tregs, defined by the markers CD4 and FoxP3, regulate immune responses by suppressing effector $T$ cells and are recruited to sites of action by the chemokine CCL22. Here, we demonstrate that production of CCL22 in islets after intrapancreatic duct injection of double-stranded adeno-associated virus encoding CCL22 recruits endogenous Tregs to the islets and confers long-term protection from autoimmune diabetes in NOD mice. In addition, adenoviral expression of CCL22 in syngeneic islet transplants in diabetic NOD recipients prevented $\beta$ cell destruction by autoreactive T cells and thereby delayed recurrence of diabetes. CCL22 expression increased the frequency of Tregs, produced higher levels of TGF- $\beta$ in the CD4 $4^{+} \mathrm{T}$ cell population near islets, and decreased the frequency of circulating autoreactive $\mathrm{CD8}^{+} \mathrm{T}$ cells and $\mathrm{CDB}^{+} \mathrm{IFN}-\gamma$-producing $T$ cells. The protective effect of CCL22 was abrogated by depletion of Tregs with a CD25-specific antibody. Our results indicate that islet expression of CCL22 recruits Tregs and attenuates autoimmune destruction of $\beta$ cells. CCL22-mediated recruitment of Tregs to islets may be a novel therapeutic strategy for type 1 diabetes.
\end{abstract}

\section{Introduction}

The complex pathogenesis of autoimmune diabetes in NOD mice and humans involves multiple immune components. The autoimmune response recurs when $\beta$ cells are transplanted into type 1 diabetic patients or NOD mice $(1,2)$. Tregs are primary mediators of peripheral self-tolerance and are able to suppress the proliferative and cytokine responses of other immune cells (3). Defects in Treg number and/or function may underlie spontaneous development of autoimmune diseases (4). The chemokine receptor CCR4 is highly expressed on Tregs and mediates recruitment of Tregs in vivo by its ligand, CCL22 (5). Recruitment of Tregs to human cancers producing CCL22 suppresses tumor-specific $\mathrm{T}$ cell immunity and contributes to tumor growth $(6,7)$. To determine whether CCL22-mediated recruitment of Tregs could counter the autoimmune response to $\beta$ cells in diabetes, we overexpressed CCL22 in $\beta$ cells in vivo in NOD mice and in syngeneic islet transplants.

\section{Results and Discussion}

CCL22 protects pancreatic $\beta$ cells from autoimmune attack in type 1 diabetes. To drive islet-specific expression of CCL22, we created double-stranded adeno-associated virus serotype 8 (dsAAV8) vectors containing mouse CCL22 or GFP cDNA, driven by the rat insulin promoter (dsAAV8-RIP/CCL22 and dsAAV8-RIP/GFP, respectively). One month after injection of $1.5 \times 10^{11}$ viral genomes (vg) via the pancreatic duct, CCL22 secretion was detected in islets isolated from dsAAV8-RIP/CCL22-infected mice but not controls. Infection did not impact $\beta$ cell function,

Authorship note: Joel Montane and Loraine Bischoff contributed equally to this work.

Conflict of interest: The authors have declared that no conflict of interest exists. Citation for this article: J Clin Invest. 2011;121(8):3024-3028. doi:10.1172/JCI43048. since immune-deficient (NOD/SCID) mice showed normal glucose tolerance at 2 and 4 months after injection with dsAAV8RIP/GFP (data not shown).

To determine whether $\beta$ cell-specific expression of CCL22 can delay or prevent diabetes, 8-week-old NOD mice were injected intraductally with dsAAV8-RIP/CCL22, dsAAV8-RIP/GFP, or PBS. NOD mice transduced with dsAAV8-RIP/CCL22 (CCL22-NOD mice) were protected from diabetes, with 9 out of 11 mice remaining normoglycemic for up to 160 days. All NOD mice transduced with dsAAV8-RIP/GFP (6 out of 6 mice) or injected with PBS (PBS-NOD mice) (7 out of 7 mice) developed diabetes within the first 100 days (Figure 1A). CCL22-NOD mice maintained normal glucose tolerance (Figure 1B) and circulating insulin levels (data not shown). Despite extensive insulitis in both CCL22-NOD and PBS-NOD mice (Figure 1C), CCL22 expression was associated with preservation of insulin-positive cells at 15 days, 1 month, and 3 months after intraductal injection.

CCL22 enhances influx of Tregs to islets and limits islet autoimmunity. By immunostaining, we observed an apparent increase in the number of $\mathrm{FoxP}^{+}$cells in islets of CCL22-NOD mice at 15 days, 1 month, and 3 months after injection (Supplemental Figure 1; supplemental material available online with this article; doi:10.1172/JCI43048DS1). Flow cytometric analysis showed that the frequency of FoxP3 ${ }^{+}$cells in pancreata of CCL22-NOD mice was significantly elevated by 1 month after injection and more markedly elevated at 3 months (Figure 2A). At 1 month after injection, the absolute number of $\mathrm{CD}^{+}{ }^{+} \mathrm{FoxP}^{+}$cells was increased in the pancreas (Figure 2B) but not in the pancreatic lymph node (PLN) (data not shown). No significant differences were observed in the absolute numbers of $\mathrm{CD}^{+}{ }^{+}$or $\mathrm{CD}^{+}$effector cells in either the pancreas or the PLN (data not shown). The Treg/CD4 effector $\mathrm{T}$ cell ratio was significantly elevated in PLNs of CCL22-NOD mice and showed a nonsignificant increase in pancreas at 1 month after 
A

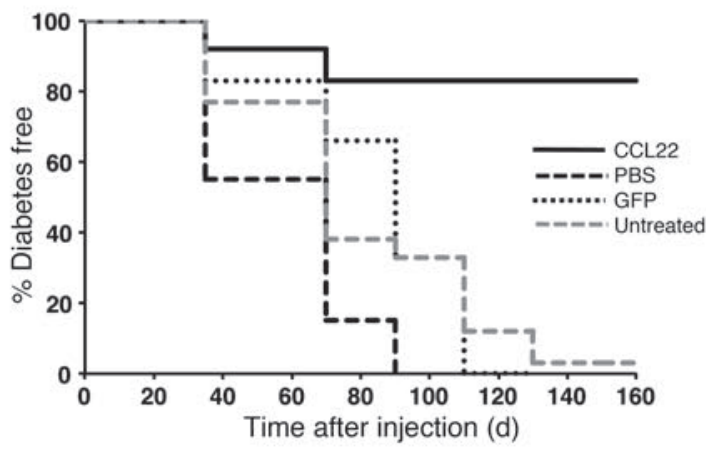

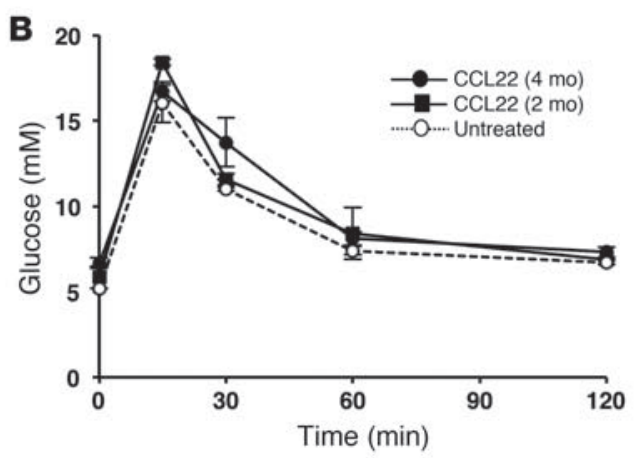
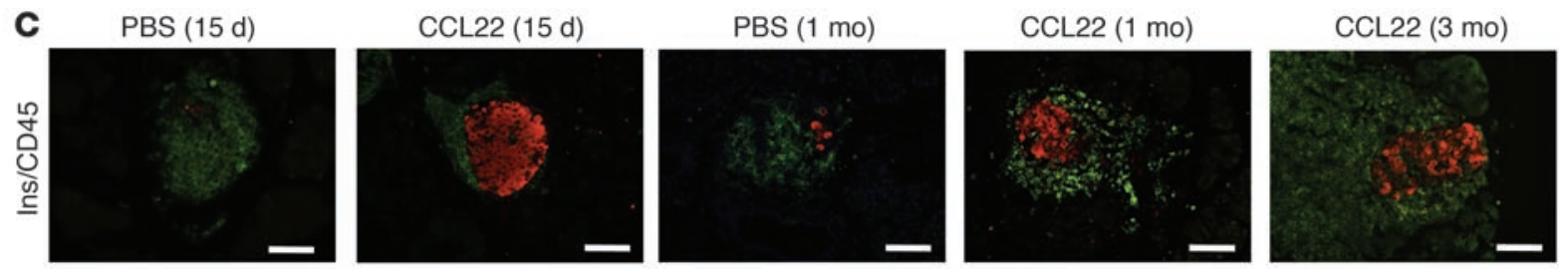

\section{Figure 1}

CCL22 expression in islets protects from diabetes development. (A) NOD females were injected at 8 weeks of age with $1.5 \times 10^{11} \mathrm{vg}$ of dsAAV8RIP/CCL22, dsAAV8-RIP/GFP, or PBS (as control) via the pancreatic duct. Kaplan-Meier survival curves were derived from blood glucose data and compared using the log-rank test $(P<0.001$ CCL22 versus PBS control; $P<0.01$ versus GFP; $P=0.001$ versus untreated). (B) i.p. glucose tolerance tests 2 and 4 months after injection of dsAAV8-RIP/CCL22 in NOD mice and untreated NOD/SCID mice as control $(n=4$ in each group). No significant difference in glucose tolerance was observed after intraductal injection and CCL22 expression. Results are expressed as mean \pm SEM. (C) Immunostaining for insulin-positive $\beta$ cells (red) and islet-infiltrating leukocytes by CD45 staining (green) of PBS- and CCL22NOD mice at indicated time points. Note the presence of intact islets containing strong insulin staining in CCL22-NOD mice compared with islets of PBS-NOD mice. Scale bar: $100 \mu \mathrm{m}$.

injection (Supplemental Figure 2). Administration of Treg-depleting (anti-CD25) antibody to CCL22-NOD mice reversed protection from diabetes (Figure 2C and Supplemental Figure 3, A and B). CCR4 expression on Tregs in the PLNs of CCL22-NOD mice appeared higher than that in PBS control mice by 1 month after injection (Supplemental Figure 4A), although this difference was not statistically significant. These data suggest that interaction of CCL22 with CCR 4 contributes to the trafficking of Tregs to both CCL22-expressing islets and the PLNs.

$\mathrm{CD}^{+}$cells in the PLN and pancreas of CCL22-NOD mice produced more TGF- $\beta$ (Figure 2D), suggesting that local CCL22 expression may suppress autoimmunity in part by increasing TGF- $\beta$ production. CCL22-NOD mice did not differ significantly from PBS-NOD controls in the proportion of $\mathrm{CD}^{+}$cells in pancreatic infiltrates at 1 month after injection; however, the percentage of IFN- $\gamma$-producing $\mathrm{CD}^{+}$cells was lower in CCL22NOD mice (Figure 2E), suggesting that islet CCL22 expression results in decreased activity of effector T cells. Quantification of autoreactive $\mathrm{CD}^{+} \mathrm{T}$ cells in blood predicts the onset of diabetes (8), and deletion of these cells can prevent diabetes in NOD mice (9). Using MHC class I tetramers specific for the $\beta$ cell autoantigens insulin and islet-specific glucose 6-phosphatase-related protein (IGRP), we found that the frequency of IGRP-reactive T cells in peripheral blood was decreased in CCL22-NOD mice compared with that in controls at 15 days and 2 months after injection. Circulating insulin-reactive $\mathrm{T}$ cells also appeared lower (Figure $2 \mathrm{~F}$ ), although this difference was not statistically significant. Taken together, these results suggest that CCL22-mediated recruitment of Tregs to islets limits expansion and effector activity of autore- active T cells. Although transgenic expression of CCL22 in islets from birth accelerates diabetes onset in NOD mice (10), we demonstrate here that AAV8-mediated, $\beta$ cell-specific expression of CCL22, starting from 8 weeks of age in vivo protects $\beta$ cells from autoimmune destruction.

CCL22 protects from recurrent autoimmunity in NOD mice. To determine whether islet CCL22 expression prevents $\beta$ cell loss in syngeneic islet transplants in NOD mice, we created an adenovirus (Ad-CCL22) in which expression of a mouse CCL22 cDNA is driven by the CMV promoter. Transduction with Ad-CCL22 (MOI of 10) resulted in islet expression of CCL22 without affecting viability or function in vitro (data not shown). Diabetic NOD female mice were transplanted with 500 syngeneic (NOD/SCID) islets transduced ex vivo with either Ad-CCL22 or Ad-LacZ or left untreated. Survival of syngeneic islet grafts in diabetic NOD recipients was markedly improved in mice transplanted with CCL22-expressing islets (Figure 3A). Protection from recurrent autoimmunity was temporary, as hyperglycemia returned in all recipients of CCL22-expressing grafts by 60 days after transplantation, likely due to gradual loss of adenoviral CCL22 expression (Supplemental Figure 5). Immunostaining revealed many insulin-producing $\beta$ cells in CCL22-expressing grafts but virtually none in controls (Supplemental Figure 6A). Islet CCL22 expression appeared to similarly protect $\alpha$ cells (Supplemental Figure 6A). Ad-CCL22-transduced grafts had a greater number of FoxP3 ${ }^{+}$cells than controls, as demonstrated by both flow cytometry (Figure 3B) and immunostaining (Supplemental Figure 6, B and C). Moreover, the ratio of Tregs to CD4+ effector cells was significantly greater in CCL22-expressing grafts (Figure 3C). 

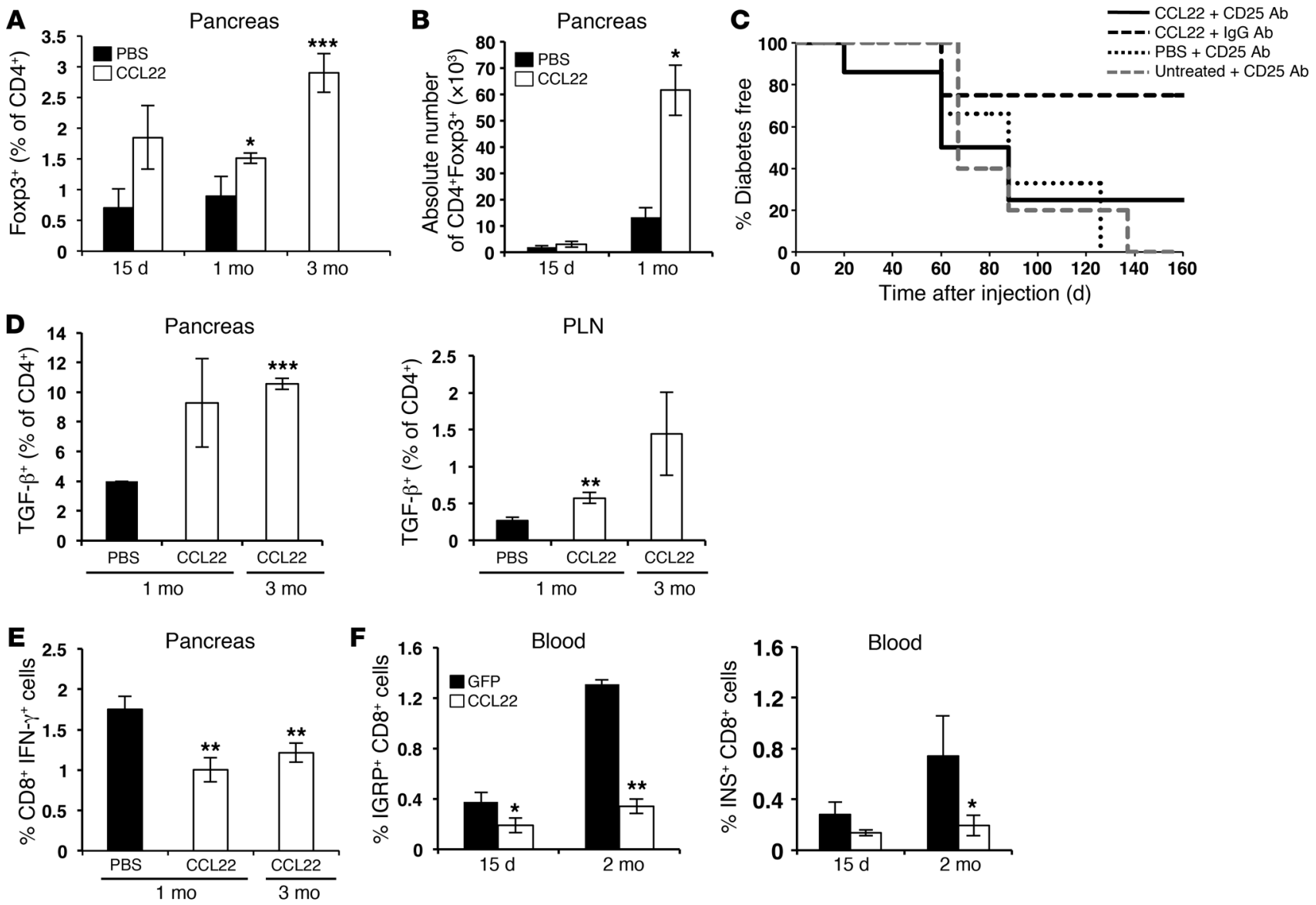

Figure 2

CCL22 expression recruits Tregs to pancreatic islets and reduces autoimmune response. (A, B, and D-F) Flow cytometric analysis of cells from CCL22-NOD and control mice. (A) The percentage of CD4+ cells that are FoxP3 ${ }^{+}$in pancreata of CCL22-NOD mice was not significantly elevated compared with that of PBS-NOD mice at 15 days but significantly increased by 1 month and further increased at 3 months after injection. (B) The absolute number of CD4+Foxp3 ${ }^{+}$cells was not significantly different at 15 days but was higher in pancreata of CCL22-NOD mice compared with that in PBS-NOD mice 1 month after injection. (C) In CCL22-NOD mice, injection of anti-CD25 antibody resulted in more rapid and frequent diabetes development than injection of isotype-matched IgG (CCL22-NOD plus anti-CD25 vs. CCL22-NOD plus IgG; $P=0.01$ ) and was not significantly different from that in NOD mice that received anti-CD25 antibody alone or with PBS. (D) Increased frequency of TGF- $\beta^{+}$ cells among CD4+ cells in CCL22-NOD mouse pancreata (3 months) and PLNs (1 month). (E) Decreased frequency of CD8 ${ }^{+}$IFN- $\gamma^{+}$cells in pancreata from CCL22-NOD mice at 1 and 3 months after injection compared with that of PBS-NOD mice at 1 month. (F) The frequency of IGRP$(n=7)$ and insulin (INS)- $(n=5)$ specific CD8 + T cells was lower in CCL22-NOD mice in peripheral blood 15 days and 2 months after injection. The frequency of total $\mathrm{CD} 8^{+}$cells in the blood was not significantly different among CCL22-NOD and control groups (not shown). ${ }^{*}<<0.05$, ${ }^{\star \star} P<0.01,{ }^{\star \star *} P<0.001$ versus PBS.

More Tregs than effector $\mathrm{CD} 4^{+}$cells were CCR4-positive in both CCL22- and LacZ-expressing grafts, and Tregs present in CCL22expressing grafts expressed higher levels of CCR4 than Tregs in controls (Supplemental Figure 4B). IGRP-reactive T cells were found in similar frequencies in both groups (Figure 3D), indicating that CCL22 expression does not prevent migration of autoreactive $\mathrm{CD}^{+}$cells to the graft. Administration of anti-CD25 antibody reversed protection from recurrent autoimmunity in recipients of CCL22-expressing islet transplants (Figure 3E). These results suggest that CCL22-mediated recruitment of Tregs decreases graft failure and recurrence of autoimmune diabetes.

In summary, recruitment of Tregs to islets by CCL22 expression delays both spontaneous and recurrent autoimmune diabetes in NOD mice. Although insulitis clearly persists in the face of CCL22mediated protection from diabetes, we speculate that CCL22 may not only enhance recruitment but also functionally activate Tregs that are optimally positioned for suppression of relevant targets (as suggested by increased CTLA-4 expression after CCL22 treatment; Supplemental Figure 7). CCL22 likely recruits CCR4-expressing effector cells, perhaps increasing the opportunity for Treg/effector cell interaction. Although islet CCL22 expression did not reverse disease in diabetic mice (data not shown), it will be of interest to assess the ability of other chemokines, such as CCL17, which also interacts with CCR4 (5), to prevent or cure diabetes. Recruitment of endogenous Tregs to the site of inflammation may obviate the need for expansion and infusion of cells for Treg-based diabetes therapies (11-13). Manipulating the migration of endogenous Tregs by gene- or peptide-delivery approaches may be a novel therapeutic strategy not only for diabetes but for other autoimmune diseases and organ transplantation. 

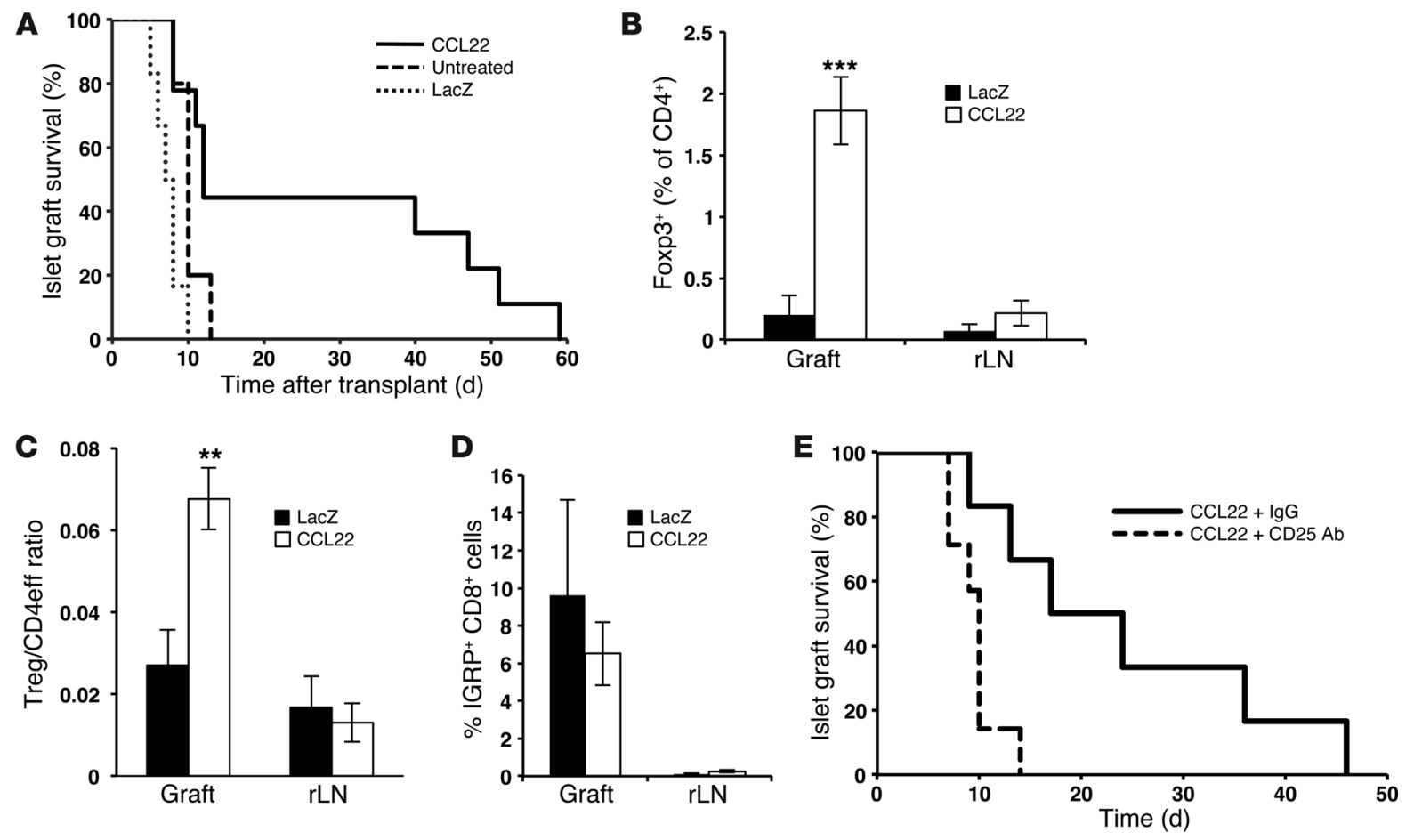

\section{Figure 3}

CCL22 expression prevents recurrent autoimmunity in NOD mice after syngeneic islet transplantation. (A) Diabetic NOD mice were transplanted with Ad-CCL22 ( $n=9)$, Ad-LacZ $(n=6)$, or untreated islets as controls $(n=5)$. Kaplan-Meier survival curves were derived from blood glucose data and compared using the log-rank test $(P<0.01$ versus untreated and LacZ). (B) Flow cytometric analysis of draining renal lymph nodes (rLNs) and islet grafts of CD4 ${ }^{+}$FoxP3 ${ }^{+}$cells (CCL22, $n=7$; LacZ, $\left.n=3\right) 10$ days after transplantation. ${ }^{* * *} P<0.001$. (C) The ratio of CD4 ${ }^{+}$FoxP3 ${ }^{+}$ Tregs to CD4+ FoxP3- effector cells (Treg/CD4eff ratio) was determined in the grafts and renal lymph nodes of CCL22 $(n=15)$ and LacZ $(n=6)$ recipients 10 days after transplantation. ${ }^{\star *} P<0.01$. (D) Detection of IGRP-specific CD8 ${ }^{+} T$ cells in islet grafts and renal lymph nodes from recipients of CCL22-expressing $(n=7)$ and LacZ-expressing $(n=2)$ grafts. $P=$ NS. (E) Depletion of Tregs in recipients of CCL22-expressing grafts using anti-CD25 antibody (anti-CD25, $n=7 ; \mathrm{IgG}, n=6$ ). Survival curves were compared using the log-rank test. $P=0.01$.

\section{Methods}

Viral vector production and amplification. Recombinant dsAAV8 vectors were generated at the Children's Hospital of Philadelphia by triple transfection protocol as previously described (14). The dsAAV8-RIP/GFP plasmid was provided by Paul Robbins (University of Pittsburgh, Pittsburgh, Pennsylvania, USA). CCL22 cDNA was from Invitrogen. To generate the adenoviral vector Ad-CCL22, pCMV/CCL22 cDNA was cloned in pShuttle $^{+/+}$plasmid. Adenovirus was produced by cotransfection of HEK293 cells with the CCL22 vector and the Adenovirus helper plasmid (ABM Good). Adenovirus was purified with the Vivapure Adenopack $100 \mathrm{Kit}$ (VivaScience) and titrated by the end-point dilution method of tissue culture infective dose. Ad-LacZ was provided by R.G. Korneluk (Children's Hospital of Eastern Ontario Research Institute, Ottawa, Ontario, Canada). The endotoxin content of the adenoviral vectors was less than 1 endotoxin units $/ \mathrm{ml}(\mathrm{EU} / \mathrm{ml})$.

Viral vector administration and islet transplantation. NOD and NOD/SCID mice were purchased from The Jackson Laboratory and housed in the Child and Family Research Institute animal facility. Animal studies were reviewed and approved by the University of British Columbia Committee on Animal Care. Injection into the pancreatic duct was performed as described previously (15). For islet transplantation, pancreatic islets were isolated from male NOD/SCID mice by ductal collagenase (Sigma-Aldrich) injection, digestion, and purification with the filtration method as described previously (16). Islets were hand picked and incubated in complete RPMI 1640 (Gibco) with Ad-CCL22 or Ad-LacZ (MOI of 10) overnight at $37^{\circ} \mathrm{C}$. Islets were transplanted into the renal subcapsular space of recipient mice under isoflurane anaesthesia. Graft failure was determined when blood glucose was greater than $15 \mathrm{mM}$ on 2 consecutive days. In some experiments, CD25 antibody (PC61; AdLab, University of British Columbia; $100 \mu$ g i.p.) or rat isotype control IgG (AbLab) was injected weekly after AAV8 administration or islet transplantation.

Islet function. In vivo islet function was assessed by i.p. glucose tolerance test. Mice were fasted for 5 hours and injected with D50 glucose $(2 \mathrm{~g} / \mathrm{kg}$ body weight; Baxter Healthcare), and tail blood glucose was measured at $0,15,30,60$, and 120 minutes.

CCL22 protein determination. CCL22 expression in islets was determined by Western blot. Ten micrograms of protein from islet lysates were resolved on a $20 \%$ SDS-polyacrylamide gel at $100 \mathrm{~V}$. After transfer to Immobilon-P membranes (Millipore) at $100 \mathrm{~V}$ for 1 hour using the Trans-Blot System (Bio-Rad), immunoblot analysis was performed using polyclonal antiCCL22 antibody (R\&D Systems) and detected by chemiluminescence (Amersham). CCL22 protein levels were measured by ELISA (R\&D Systems) following the manufacturer's instructions.

Histological analysis of the pancreas. Pancreata were fixed in $4 \%$ paraformaldehyde overnight. Paraffin sections (5- $\mu \mathrm{m}$ thick) were immunostained using guinea pig anti-insulin and rabbit anti-glucagon antibodies (Dako) as well as goat anti-guinea pig Alexa Fluor 594 and goat anti-rabbit Alexa Fluor 594 (Molecular Probes) as secondary antibodies. Leukocyte immunostaining was performed after antigen retrieval by steaming for 30 minutes in Dako Target Retrieval Solution, using rat anti- 
mouse CD45 antibody (BD Pharmingen) or anti-mouse FoxP3 antibody (eBioscience) and anti-rat Alexa Fluor 488 secondary antibody (AdLab, University of British Columbia). Images were captured using an Olympus Bx61 microscope and In Vivo or DP software.

Flow cytometry. Pancreata were excised and digested with $1,000 \mathrm{U} / \mathrm{ml}$ of collagenase (Sigma-Aldrich) in Hank's buffer solution (Life Technologies) for 20 minutes at $37^{\circ} \mathrm{C}$. The resulting cell homogenate was filtered through a $40-\mu \mathrm{m}$ filter basket and washed in PBS. Harvested grafts and lymph nodes were strained through a $40-\mu \mathrm{m}$ filter and washed before incubation with antibodies. Monoclonal antibodies to CD8 (53-6.7) and CD4 (RM4-5) were purchased from BD Biosciences. Antibodies to FoxP3 (clones MF-14 and 150D) and CCR4 (clone 2G12) were obtained from BioLegend. Antibodies to IFN- $\gamma$ (XMG1.2) and CTLA4 were from eBioscience, and antibody to antiTGF- $\beta$ (1D11) was from R\&D Systems. Tetramers with IGRP $206-214$ or Insulin $\mathrm{B}_{15-23}$ epitopes were generated and provided by the Cellular Immunology Core facility at the Child and Family Research Institute. For intracellular staining, cells were fixed and permeabilized using the Cytofix/Cytoperm Kit (BD Biosciences). For intracellular cytokine staining, cells were stimulated in culture medium containing $100 \mathrm{ng} / \mathrm{ml} \mathrm{PMA}, 1 \mu \mathrm{g} / \mathrm{ml}$ ionomycin, and $10 \mu \mathrm{g} / \mathrm{ml}$ brefeldin A (Sigma-Aldrich) at $37^{\circ} \mathrm{C}$ for 4 hours. Absolute cell number was determined by adding 50,000 counting beads (CountBright, Invitrogen) per sample before flow cytometry. A FACSCalibur cytometer (BD Biosciences) and CellQuest software (BD Biosciences) were used for flow cytometry, and data were analyzed using FlowJo 7.2.5 software.

Statistics. Graft survival curves were generated using Kaplan-Meier lifetable analysis and were compared using the log-rank test. Other statisti- cal analyses were performed using Student's 2 -tailed $t$ test. $P<0.05$ was considered statistically significant. Data in bar graphs are represented as mean \pm SEM.

\section{Acknowledgments}

This work was supported by grants from Canadian Institute of Health Research (CIHR; RMF-79029 and MOP-64427), Juvenile Diabetes Research Foundation, and Michael Smith Foundation for Health Research (MSFHR) to T.J. Kieffer, C.B. Verchere, and R. Tan. C.B. Verchere, T.J. Kieffer, and R. Tan are senior scholars of the MSFHR. M.K. Levings holds a Canada Research Chair in Transplantation. J. Montane and L. Bischoff are supported by trainee awards from the CIHR Transplant Training Program, the Child and Family Research Institute, and the Juvenile Diabetes Research Foundation. G. Hardenberg is supported by a fellowship from MSFHR. We thank the Viral Vector Core of the Children's Hospital of Philadelphia for generating the viral vectors and members of the Verchere laboratory for helpful discussion.

Received for publication March 19, 2010, and accepted in revised form May 11, 2011.

Address correspondence to: Bruce Verchere, Child and Family Research Institute, 950 West 28th Ave., Vancouver, British Columbia, V5Z 4H4, Canada. Phone: 604.875.2490; Fax: 604.875.2373; E-mail: bverchere@cfri.ca.
1. Sutherland DE, Goetz FC, Sibley RK. Recurrence
of disease in pancreas transplants. Diabetes. 1989;
38 suppl 1:85-87.
2. Trudeau JD, Chandler T, Soukhatcheva G, Verchere
CB, Tan R. Prospective prediction of spontane-
ous but not recurrent autoimmune diabetes in
the non-obese diabetic mouse. Diabetologia. 2007 ;
50(5):1015-1023.
3. Vignali DA, Collison LW, Workman CJ. How regulato-
ry T cells work. Nat Rev Immunol. 2008;8(7):523-532.
4. Sgouroudis E, Piccirillo CA. Control of type 1 diabe-
tes by CD4+Foxp3+ regulatory T cells: lessons from
mouse models and implications for human disease.
Diabetes Metab Res Rev. 2009;25(3):208-218.
5. Iellem A, et al. Unique chemotactic response pro-
file and specific expression of chemokine receptors
CCR4 and CCR8 by CD4(+)CD25(+) regulatory T
cells. J Exp Med. $2001 ; 194(6): 847-853$.
6. Curiel TJ, et al. Specific recruitment of regula- tory $\mathrm{T}$ cells in ovarian carcinoma fosters immune privilege and predicts reduced survival. Nat Med. 2004;10(9):942-949.

7. Qin XJ, Shi HZ, Deng JM, Liang QL, Jiang J, Ye ZJ. CCL22 recruits CD4-positive CD25-positive regulatory $\mathrm{T}$ cells into malignant pleural effusion. Clin Cancer Res. 2009;15(7):2231-2237.

8. Trudeau JD, et al. Prediction of spontaneous autoimmune diabetes in NOD mice by quantification of autoreactive $\mathrm{T}$ cells in peripheral blood. $J$ Clin Invest. 2003;111(2):217-223.

9. Han B, et al. Prevention of diabetes by manipulation of anti-IGRP autoimmunity: high efficiency of a low-affinity peptide. Nat Med. 2005;11(6):645-652.

10. Kim SH, Cleary MM, Fox HS, Chantry D, Sarvetnick $\mathrm{N}$. CCR4-bearing $\mathrm{T}$ cells participate in autoimmune diabetes. J Clin Invest. 2002;110(11):1675-1686.

11. Brusko TM, Bluestone JA. Regulatory T cells directed to the site of the action. Proc Natl Acad Sci U S A.
2009;106(49):20553-20554.

12. Goudy KS, et al. Systemic overexpression of IL-10 induces CD $4+C D 25+$ cell populations in vivo and ameliorates type 1 diabetes in nonobese diabetic mice in a dose-dependent fashion. J Immunol. 2003; 171(5):2270-2278.

13. Tang $Q$, et al. In vitro-expanded antigen-specific regulatory $\mathrm{T}$ cells suppress autoimmune diabetes. J Exp Med. 2004;199(11):1455-1465.

14. Matsushita T, et al. Adeno-associated virus vectors can be efficiently produced without helper virus. Gene Ther. 1998;5(7):938-945.

15 . Wang $Z$, et al. Widespread and stable pancreatic gene transfer by adeno-associated virus vectors via different routes. Diabetes. 2006;55(4):875-884.

16. Salvalaggio PR, et al. Islet filtration: a simple and rapid new purification procedure that avoids ficoll and improves islet mass and function. Transplantation. 2002;74(6):877-879. 